\title{
Editorial special issue on "Robust and stochastic optimization in production systems and supply chain management"
}

\author{
Laureano F. Escudero • Stefan Minner
}

Published online: 2 November 2013

(C) Springer-Verlag Berlin Heidelberg 2013

Uncertainty, disruptions, and variability are main challenges of manufacturing systems and supply chains. The design and operation of such systems have to incorporate uncertainty about the future. Adaptability and flexibility are desirable features of such systems, as are robust designs and plans. Operations research methods support strategic and operational decisions in production and supply chains and the evaluation of different concepts. However, there exist several different uncertainty modelling approaches that make different assumptions about the knowledge on availability and estimation of the required distributions and parameter values for optimization. This specially contains applied contributions on uncertainty modelling and optimization in the fields of logistics, manufacturing, and supply chain management where robust and/or stochastic models are used to provide decision support.

This special issue was inspired by focused topics of the APMOD conference 2012 held at the University of Paderborn. Several manuscripts presented at the conference were submitted in response to an open call for papers on the topic. The following 5 selected manuscripts present applications of stochastic programming and robust optimization of several different industries and fields of application.

Alem and Morabito, "Risk-averse two-stage stochastic program in furniture plants" present a case application from the furniture industry and use two-stage mixed-integer programming formulations under different decision criteria taking risk into account.

\footnotetext{
L. F. Escudero

Departamento de Estadística e Investigaciǿn-Operativa, Universidad Rey Juan Carlos, c/ Tulipan s/n, 28933 Mostoles, Madrid, Spain e-mail: laureano.escudero@urjc.es

S. Minner $(\bowtie)$

TUM School of Management, Technische Universität München, Arcisstraße 21, 80333 Munich, Germany

e-mail: stefan.minner@tum.de
} 
The authors show that the use of these criteria can significantly reduce risk without too much compromising on expected performance. Schöneberg, Koberstein and Suhl, "A stochastic programming approach to determine robust delivery profiles in area forwarding inbound logistics networks" present a two-stage stochastic mixedinteger linear programming model to determine robust delivery profiles for consolidating supplies. A solution framework with scenario generation, decomposition, a genetic algorithm, and a standard MIP-solver is proposed and an industry case application is presented. Kawas, Laumanns and Pratsini, "A robust optimization approach to enhancing reliability in production planning under non-compliance risks" investigate a production planning problem under uncertainty in a game-theoretic setting with an inspection agency that operates under product quality uncertainty. The company's failure to comply with regulatory requirements impacts supply delivery. In order to solve the model and to provide managerial insights, a robust, mixed-integer linear programming approach is presented. Klibi and Martel, "The design of robust value-creating supply chain networks" present a supply chain design problem under uncertainty with a strategic and an operational stage including the option to make structural changes. A Monte-Carlo sampling and sample average approximation solution strategy are proposed and used in an illustrative case for a location-transportation problem. Bozorgi-Amiri, Jabalameli and Mirzapour-Al-e-Hashem, "A multi-objective robust stochastic programming model for disaster relief logistics under uncertainty" present a stochastic programming approach with multiple criteria for a humanitarian disaster relief problem with several sources of parameter uncertainty. This is applied in an earthquake case study scenario in Iran.

Laureano F. Escudero, Stefan Minner (Guest Editors) 